\title{
Claude Ghiati, Isabelle Havelange, Brigitte Keriven et Martine Sonnet, Bibliographie annuelle de l'histoire de France du cinquième siècle à 1958.
}

\section{Bernard Merdrignac}

\section{(2) OpenEdition \\ 1 Journals}

Édition électronique

URL : http://journals.openedition.org/abpo/1404

DOI : $10.4000 /$ abpo.1404

ISBN : 978-2-7535-1491-1

ISSN : $2108-6443$

Éditeur

Presses universitaires de Rennes

\section{Édition imprimée}

Date de publication : 20 octobre 2003

Pagination : 141-142

ISBN : 978-2-86847-929-7

ISSN : $0399-0826$

\section{Référence électronique}

Bernard Merdrignac, «Claude Ghiati, Isabelle Havelange, Brigitte Keriven et Martine Sonnet,

Bibliographie annuelle de l'histoire de France du cinquième siècle à 1958. ", Annales de Bretagne et des Pays de l'Ouest [En ligne], 110-3 | 2003, mis en ligne le 20 décembre 2005, consulté le 23 septembre 2020.

URL : http://journals.openedition.org/abpo/1404; DOI : https://doi.org/10.4000/abpo.1404 
centrale, il s'arrêta à la première étape en exaltant une nation bretonne idéale mais il ne fonda pas d'organisation et ne milita pas, d'abord parce que pour lui la nation bretonne s'était évanouie et appartenait au passé. Il se voulait chantre de cette patrie bretonne et totalement fidèle à la grande patrie, la France. On le comprend, les mouvements nationalistes ne purent en faire vraiment leur héraut et se trouvaient en porte à faux face à sa pensée.

Les communications font le point sur les travaux et l'action de La Borderie et c'est déjà beaucoup, même si les avis divergent quelque peu, mais on peut trouver plus dans ce livre : un homme, un véritable intellectuel d'une ampleur indiscutable et donc l'action multiforme imprima sa marque à son époque. Un portrait émane des différents articles, à commencer par ceux qui sont explicitement centrés sur l'aspect biographique, la grande synthèse initiale de J. Gury ou celui que $\mathrm{X}$. Ferrieu consacre à sa jeunesse et à ses années de formation. La Borderie apparaît comme profondément inscrit dans son siècle. C'est un bourgeois aisé, capable de vivre de ses rentes et de se consacrer à ses études en toute liberté. C'est aussi un conservateur, monarchiste mais relativement modéré. En notable, il abordera la carrière politique qui le mènera à la députation au début de la III ${ }^{\mathrm{e}}$ République mais sans grande passion. Par contre, il se révèle un animateur hors pair qui fonde et anime associations et sociétés savantes, qui mène le débat dans de multiples congrès et atteint une position dominante dans le travail historique en Bretagne. Enfin, au fil des pages se dévoile ce qu'était l'histoire selon lui et c'est à une passionnante recherche d'historiographie que nous sommes conviés. Ainsi revit, une aventure intellectuelle, une étape de la recherche historique et l'on peut regretter que l'historien ne soit jugé que par rapport à notre temps, ce qui lui vaut évidemment de graves reproches mais il n'est évoqué que trop occasionnellement ce qu'il apporta par rapport à ses prédecesseurs et qui ne fut pas mince.

Au-delà du bilan, indispensable bien sûr, de l'historien ce volume nous apporte le portrait très fouillé d'un intellectuel majeur du xIX ${ }^{\mathrm{e}}$ siècle breton.

Daniel PICHOT

Ghiati, Claude, Havelange, Isabelle, Keriven, Brigitte, Sonnet, Martine, Bibliographie annuelle de l'histoire de France du cinquième siècle à 1958. Année 2001, t. 47, CNRS éditions, Paris, 2002, 1080 p.

La Bibliographie annuelle de l'histoire de France qui rassemble chaque année depuis 1953 les articles et ouvrages français et étrangers concernant l'histoire de France a rendu un demi-siècle de bons et loyaux services à la communauté des historiens. Entre-temps, avec l'aide constante de la Bibliothèque (puis de la BNF), les ingénieures du CNRS responsables de l'ouvrage sont passées des fiches manuscrites à la saisie informatisée des données. Cette quarante-septième livraison de l'ouvrage rassemble 13085 notices provenant des dépouillements de 1287 revues françaises et 729 revues étrangères auxquels s'ajoutent 282 ouvrages collectifs (mélanges, actes de colloque, etc.). Le classement formel des références en neuf grandes rubriques qui reflète les principales directions de la recherche historique a bien entendu été conservé (" manuels généraux et sciences auxiliaires de l'histoire "; " histoire politique "; « histoire des institutions "; « histoire économique "; " histoire sociale "; " histoire religieuse "; " histoire de la France outre-mer "; " histoire de la civilisation "; « histoire locale "). 
Les conditions de préparation de l'ouvrage - alors même que les CNRS-éditions envisagent l'éventualité de la mise en ligne de ces données - n'ont pas permis la présentation des statistiques historiographiques habituelles. La reprise de l'" avertissement " liminaire dans lequel les auteurs commentent celles-ci est annoncée pour la prochaine livraison. On trouvera toutefois les tableaux publiés dans le tome 46 qui constituent un observatoire privilégié des oscillations de la recherche historique sur le site internet de l'Institut d'histoire moderne et contemporaine. Ce dernier comporte en effet une page consacrée à la $B A H F$ - http://www.ihmc.ens.fr/BAHF.html - dont la consultation est fort instructive. Pour ne tirer qu'une seule information des commentaires qu'appellent ces données, constatons que l'histoire économique, l'histoire sociale, l'histoire de l'enseignement, l'histoire des institutions, l'histoire religieuse, celle des sciences et celle du livre se distinguent par leur production abondante (articles français et étrangers confondus).

Quoi qu'il en soit de l'évolution prévisible du support de la $B A H F$ qui présenterait le double avantage subsidiaire d'être évolutif et ainsi mieux adapté à l'évolution des conditions de travail des historiens, l'affirmation sur ce site de la nécessité de poursuivre simultanément la publication des volumes imprimés est réconfortante. La pérennité de la version-papier de cet indispensable instrument de travail est gage de conservation à long terme et du confort de la consultation par les utilisateurs habituels. Bien entendu dans le présent volume comme dans les précédents, un triple index (index chronologique, index-matière et table des noms d'auteurs) met plus de trois cents pages au service du lecteur. Quelques améliorations ont toutefois été apportées. Dans l'index-matière, l'ordre alphabétique est désormais discontinu. De plus, le classement alphabétique des titres exclut enfin la prise en compte des articles, ce qui permet de se repérer plus facilement dans le cas des ouvrages collectifs (" Mélanges et Actes de congrès "). En consultant ce bel ouvrage, le plaisir de feuilleter demeure intact; s'y ajoute souvent pour le chercheur, celui de découvrir, par surcroît, ce qu'il ne s'attendait pas forcément à y trouver.

Bernard MERDRIGNAC 